\title{
Determine of Midwifery Students Dietary and Physical Activity Attitudes and Behaviors: Analytic Study
}

\author{
Aysegul Durmaz* \\ Department of Midwifery, Kutahya Health Sciences University, Turkey
}

Submission: December 28, 2018; Published: January 11, 2019

*Corresponding author: Department of Midwifery, Kutahya Health Sciences University, Turkey

\begin{abstract}
Background: The less physical activity and an unhealthy diet are the factors contributing to the poor health among university students. This study aims to address this, by investigating the factors that influence eating behaviour and attitudes.

Methods: This is an analytic study. The study was carried out on May 2018 in Kutahya, Turkey. The research was completed with the participation of 333 students. The Diet-Exercise Behaviour Scale (DEBS) and The Diet-Exercise Attitude Scale (DEAS) were used to determine the diet behaviours and attitudes of the students.

Results: This is an analytic study. The study was carried out on May 2018 in Kutahya, Turkey. The research was completed with the participation of 333 students. The Diet-Exercise Behaviour Scale (DEBS) and The Diet-Exercise Attitude Scale (DEAS) were used to determine the diet behaviours and attitudes of the students.

Conclusion: Habitual physical activity, regular and balanced diet established during the university years may provide the greatest likelihood of impact on morbidity and longevity. Efforts should be focused on facilitating an active lifestyle for a person in an effort to put a stop to the increasing prevalence of obesity.
\end{abstract}

Keywords: Dietary; Physical Activity; Attitudes; Behaviors

Abbrevations: DEAS: Diet-Exercise Attitude Scale; DEBS: Diet-Exercise Behaviour Scale

\section{Introduction}

A healthy diet is crucial for the growth and the development, that contributed to prevent future illness and improve the quality and the length of the life [1]. The World Health Organization (WHO) addresses two of the major risk factors which are responsible for the heavy and growing burden. Up to $60 \%$ of the global deaths and almost half (47\%) of the global burden disease have been related to an unhealthy diet and physical inactivity. Poor eating and physical inactivity are associated with the higher health risks that are high blood pressure, elevated blood cholesterol, low intake of fruits and vegetables and high body mass index [2].

The initiating to university has been identified as an anxiety period of transition for many young adults. For instance; the first time living away from the home causes to the high rates of unhealthy behaviours which are common in this particular population. The less physical activity and an unhealthy diet are the factors contributing to the poor health among university students. Therefore, university students are considered as a risk group $[3,4]$. The recent studies about eating disorders were focused mainly on adolescents [5]. The other important strategy is the reducing excessive sedentary behaviour [6,7]. The sedentary lifestyle automatically leads to the obesity. The regular physical activity fixed during the early life years may provide the greatest likelihood of impact on the mortality and the longevity [8]. Midwives are in a unique position, given the emphasis of the philosophy of midwifery care on incorporating a holistic approach with the aim of promoting health and preventing illness [9]. Therefore, this specific population is important because of the support for the health promotion. This study was aimed to determine the eating and the physical activity behaviours and the attitudes in midwifery students.

\section{Materials and Methods}

\section{Study design and ethical considerations}

This is an analytic study. The study was carried out on May 2018 in Kutahya, Turkey. The target population of this study was midwifery student. 444 students of the Department of Midwifery of 2017-2018 academic year formed the research sample. All students were planned to include the sampling. The research was completed with the participation (participation rate 75\%) of 333 
students. The study was approved by The Institution and Ethics Committee and carried out in accordance with the Declaration of Helsinki. The participants signed an informed consent.

\section{Participants}

Inclusion criteria: Those who voluntarily agree to participate in the research after being informed by the researchers. Fully fill in the questions of the Diet-Exercise Attitude Scale and DietExercise Behaviour Scale. The participants who can completely fill the questions of the scales and who can understand, read and write in Turkish are included in the research.

Measures: Data collection: the basic information questionnaire form, diet-exercise attitude scale and diet-exercise behaviour scale were used. The basic information questionnaire included information about age, height, weight, residence, family type, family history of obesity and dietary history.

The Diet-Exercise Attitude Scale (DEAS) was used to determine the attitudes of the students towards diet. The DEAS which was adapted from the work of Yurt (2015), consisted of 13 items with Likert responses (Each item uses five points Likert type ranging from "always" to "never". A score of five points was given for "always", four points for "usually", three points for "sometimes", two points for "rarely," and one for "never"), and were summed to create a score ranging from 13 to 65 . All items in the scale contain positive expression. The higher is the score, the better is the attitude at diet and exercise behaviours [10].

The Diet-Exercise Behaviour Scale (DEBS) was used to determine the nutritional behaviours of the students. The DEBS comprise 45 items with Likert responses, and is summed to create a score ranging from 45 to 225 . Items $7,8,9,10,11,12$, $14,15,17,18,20,22,30,31,32,34,35,36,37,38,39,42,43$ are encoded in the opposite direction. The scale interpretation is based on the scores obtained from the subscales. DEBS include four subscales: 1-psychological eating, 2-healthy eating-exercise behaviour, 3-unhealthy eating-exercise behaviour, 4-meal scheme. Psychological eating consists of 11 items. The total possible score ranges from 11 to 55 (higher scores indicate that psychological eating behaviour is high.). Healthy eating-exercise behaviour consists of 14 items. The total possible score ranges from 14 to 70 (higher scores indicate that healthy eating-exercise behaviour is high). Unhealthy eating-exercise behaviour consists of 14 items. The total possible score ranges from 14 to 70 (higher scores indicate that unhealthy eating-exercise behaviour is high). Meal scheme consists of 6 items. The total possible score ranges from 6 to 30 (the higher the score, the better is the meal scheme).

\section{Statistical Analysis}

The statistical analyses were performed using IBM SPSS (Statistical Package for Social Sciences) Statistics 22 software. Descriptive statistics (mean, standard deviation, number, percentage) were used to summarize demographic data. Kolmogorov-Smirnov test was used to test the normality of distribution before the further analysis. As a result, the MannWhitney $U$ and Kruskal-Wallis Test were used for comparing variables. Correlations between the DEAS and the DEBS subscales were determined using Spearman correlation coefficient. To explain the relationship between DEBS Subscales mean and DEAS total score, linear logistic regression analysis (OR, CI, R2) was applied to the total sample. Statistical significance was set at the level of $\mathrm{p}<0.050$.

\section{Results}

Demographic and selected lifestyle characteristics of the 333 midwifery students who participated in the present study are summarized in Table 1 . The average age of all participants was $20.07 \pm 1.49$ years. The participants were generally within the range of healthy weight $(22.73 \pm 3.55)$ and physically active $(65.8 \%)$. More than half of participants (65.8\%) were in the nuclear family structure. For family monthly income it was found that $44.1 \%$ ( $n=147$ ) was answered equal to revenue expense. $45 \%$ of the participants had obesity in their family history. The frequency of fast food consumption included once a week (37.2\%). About half of participants (46.2\%) do something else (watching television (TV)/video, reading newspaper, novel, working at the desk, sitting in front of the computer, driving a car etc. while eating. A half of participants (58.6\%) eating in front of $\mathrm{TV}$, computer or smart phone screens (Table 1).

Table 1: Socio-demographic, diet and physical activity characteristics.

\begin{tabular}{|c|c|c|c|c|}
\hline Characteristics & $n=333$ & $\%$ & Mean \pm SD & Range \\
\hline Age & & & $20.07 \pm 1.49$ & $18-26$ \\
\hline BMI & & & $22.73 \pm 3.55$ & $16-37$ \\
\hline \multicolumn{3}{|l|}{ Family Type } & & \\
\hline Nuclear family & 219 & 65.8 & & \\
\hline Extended family & 90 & 27 & & \\
\hline Broken family & 24 & 7.2 & & \\
\hline \multicolumn{5}{|l|}{ Income State } \\
\hline Less than revenue expense & 115 & 34.5 & & \\
\hline Equal to revenue expense & 147 & 44.1 & & \\
\hline More than revenue expense & 71 & 21.3 & & \\
\hline
\end{tabular}




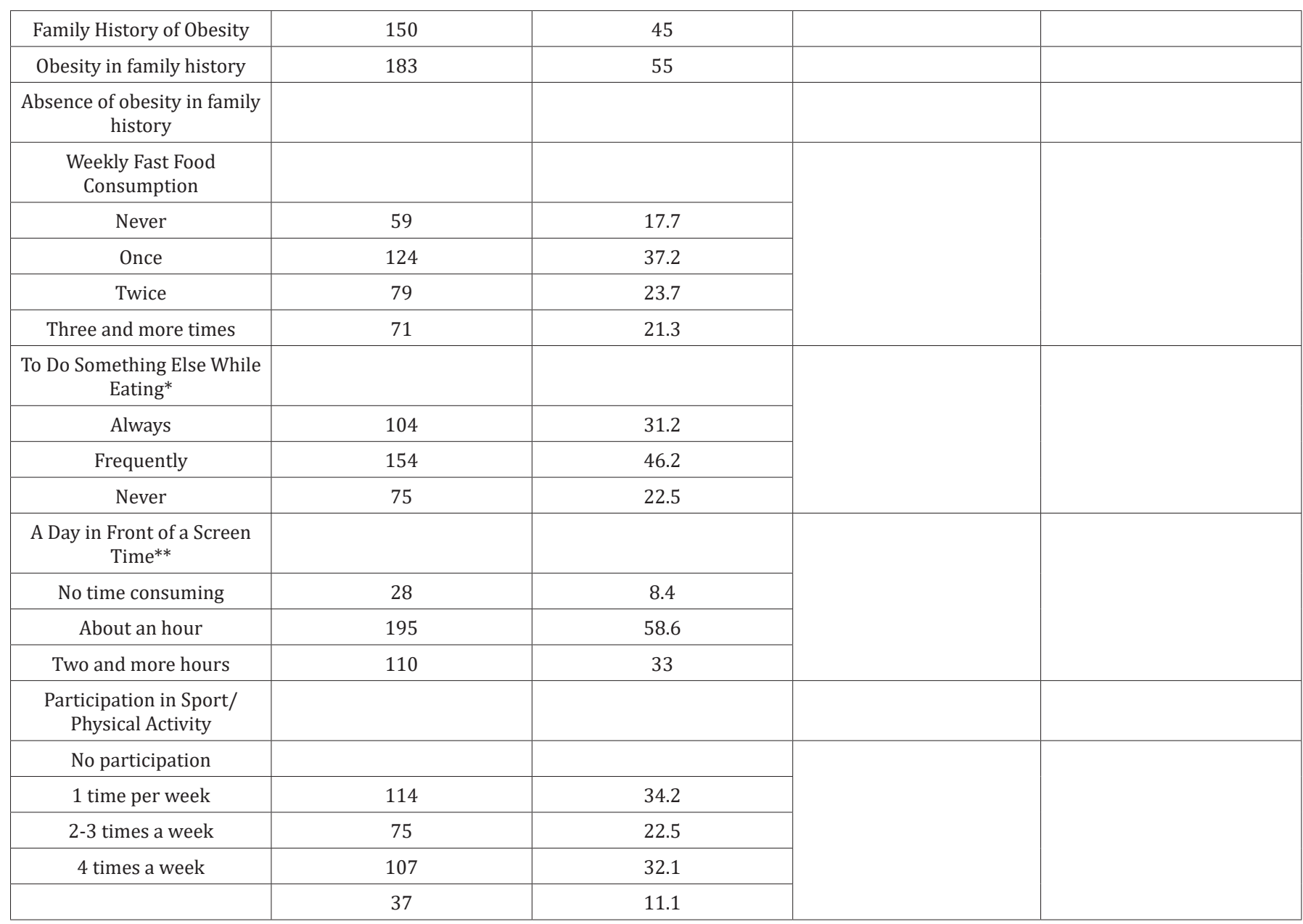

*watching TV/video, reading newspaper/book, working at the desk, sitting in front of the computer, driving a car, etc.

${ }^{* *} \mathrm{TV}$, computer or smartphone screens.

Table 2: Spearman Correlation Coefficients of DEBS Subscales Score with DEAS Total Score

\begin{tabular}{|c|c|c|c|c|c|c|}
\hline \multirow{3}{*}{ Subscales and Total } & \multicolumn{6}{|c|}{ Diet-Exercise Attitude Scale } \\
\hline & \multirow{2}{*}{$\begin{array}{l}\text { Range (Min.- } \\
\text { Max.) }\end{array}$} & \multirow{2}{*}{ Mean \pm SD } & \multicolumn{4}{|c|}{ Statistically Significant } \\
\hline & & & $\mathbf{W}^{*}$ & $\mathbf{p}^{*}$ & $\mathbf{r}^{* *}$ & $\mathbf{p}^{* *}$ \\
\hline DEAS Total & $(13-65)$ & $49.11 \pm 13.47$ & & & & \\
\hline \multicolumn{7}{|l|}{ DEBS } \\
\hline Psychological eating & $(11-55)$ & $34.62 \pm 13.18$ & 259.655 & 0 & -0.83 & 0 \\
\hline Healthy Eating-Exercise Behavior & $(14-70)$ & $48.39 \pm 16.35$ & 161.281 & 0 & 0.579 & 0 \\
\hline Unhealthy Eating-Exercise Behavior & $(17-70)$ & $43.20 \pm 15.95$ & 116.236 & 0 & -0.17 & 0.002 \\
\hline Meal Scheme & $(6-30)$ & $22.01 \pm 6.09$ & 115.397 & 0 & 0.445 & 0 \\
\hline
\end{tabular}

*Kruskal-Wallis Test, ${ }^{* *}$ Spearman correlation coefficient

The mean of the DEAS total scores was $49.11 \pm 13.47$. The mean of the DEBS's subscales scores were shown on (Table 2). The psychological eating subscale mean score was $34.62 \pm 13.18$, a healthy diet-exercise behaviour subscale mean score was $48.39 \pm 16.35$, unhealthy diet-exercise behaviour mean score was $43.20 \pm 15.95$ and meal scheme mean score was $22.01 \pm 6.09$. The
DEBS's subscales mean scores were higher than its subscales average score. There was a statistically significant difference $(\mathrm{p}<0.000)$ between the DEBS subscales (except healthy dietexercise behaviour and meal scheme) mean score and DEAS total score. The psychological eating (power correlate) and unhealthy diet-exercise behaviour (weak correlate) mean score negatively 
correlate with DEAS total score. A healthy diet-exercise behaviour and meal scheme mean score positively power correlate with DEAS total score (Table 2).

In order to examine the relationship between DEBS Subscales mean and DEAS total score, the logistic regression analysis was conducted on the total sample. In the logistic regression model, DEAS total score was used as the dependent variables. The independent variables entered into the model were DEBS subscales. In the regression, psychological eating, a healthy diet- exercise behaviour, unhealthy diet exercise behaviour and meal scheme were associated with DEAS total score. As for each unit increase in psychological eating and unhealthy diet exercise behaviour subscales, the developing positive attitude at diet and exercise behaviours decreases (OR $=-0.776,95 \%$ CI $-0.848--0.704$; OR $=-0.151,95 \%$ CI $-0.241--0.061)$. Higher scores in a healthy dietexercise behaviour and meal scheme subscales indicated more positive attitudes at diet and exercise behaviours (OR $=0.511$, 95\% CI 0.441-0.581; OR=0.878, 95\% CI 0.658-1.097) (Table 3).

Table 3: Logistic Regression Analysis of DEBS Subscales Score with DEAS Total Score.

\begin{tabular}{|c|c|c|c|c|c|}
\hline \multirow{2}{*}{ Subscales } & \multirow{2}{*}{ Odds Ratio } & \multicolumn{3}{|c|}{ Diet-Exercise Attitude Scale 95\% Confidence Interval } \\
\cline { 2 - 5 } & & Lower & Upper & $\mathbf{R}^{2}$ & \\
\hline DEBS & -0.776 & -0.848 & -0.704 & 0.576 & 0 \\
\hline Psychological eating & 0.511 & 0.441 & 0.581 & 0.385 & 0 \\
\hline $\begin{array}{c}\text { Healthy Eating-Exer- } \\
\text { cise Behavior }\end{array}$ & -0.151 & -0.241 & -0.061 & 0.032 & 0.001 \\
\hline $\begin{array}{c}\text { Unhealthy Eating-Ex- } \\
\text { ercise Behavior }\end{array}$ & 0.878 & 0.658 & 1.097 & 0.158 & 0 \\
\hline Meal Scheme & & & & 0 \\
\hline
\end{tabular}

Table 4: Association of DEBS Subscales Score, DEAS Total Score $(n=333)$ and socio-demographic, diet, physical activity characteristics.

\begin{tabular}{|c|c|c|c|c|c|c|c|c|c|c|c|c|c|c|c|}
\hline \multirow{3}{*}{$\begin{array}{l}\text { Charac- } \\
\text { teris } \\
\text {--tics }\end{array}$} & \multicolumn{3}{|c|}{$\begin{array}{c}\text { DEAS } \\
\text { Total Score }\end{array}$} & \multicolumn{3}{|c|}{$\begin{array}{l}\text { Psychological } \\
\text { eating }\end{array}$} & \multicolumn{3}{|c|}{$\begin{array}{c}\text { Healthy Eating-Exercise } \\
\text { Behavior }\end{array}$} & \multicolumn{3}{|c|}{$\begin{array}{c}\text { Unhealthy } \\
\text { Eating-Exercise } \\
\text { Behavior }\end{array}$} & \multicolumn{3}{|c|}{$\begin{array}{c}\text { Meal } \\
\text { Scheme }\end{array}$} \\
\hline & $\begin{array}{c}\text { Mean } \\
\pm \\
\text { SD }\end{array}$ & \multirow{2}{*}{$\begin{array}{l}* * \mathbf{W} \\
/ \mathbf{M}^{*}\end{array}$} & \multirow{2}{*}{$\mathbf{p}$} & $\begin{array}{c}\text { Mean } \pm \\
\text { SD }\end{array}$ & \multirow{2}{*}{$\begin{array}{l}* * \mathbf{W} \\
/ \mathbf{M}^{*}\end{array}$} & \multirow{2}{*}{ p } & $\begin{array}{l}\text { Mean } \pm \\
\text { SD }\end{array}$ & \multirow{2}{*}{$\begin{array}{l}* * \mathbf{W} \\
/ \mathbf{M}^{*}\end{array}$} & \multirow{2}{*}{$\mathbf{p}$} & $\begin{array}{c}\text { Mean } \pm \\
\text { SD }\end{array}$ & \multirow{2}{*}{$\begin{array}{l}{ }^{* *} \mathbf{W} \\
/ \mathbf{M}^{*}\end{array}$} & \multirow{2}{*}{$\mathbf{p}$} & $\begin{array}{c}\text { Mean } \\
\pm \\
\text { SD }\end{array}$ & \multirow{2}{*}{$\begin{array}{l}* * \mathbf{W} \\
/ \mathbf{M}^{*}\end{array}$} & \multirow{2}{*}{$\mathbf{p}$} \\
\hline & (Range) & & & (Range) & & & (Range) & & & (Range) & & & (Range) & & \\
\hline \multirow[t]{2}{*}{ BMI } & $\begin{array}{c}49.11 \\
\pm \\
13.47\end{array}$ & \multirow[t]{2}{*}{93.285} & \multirow[t]{2}{*}{0} & $\begin{array}{l}34.62 \\
\pm \\
13.18\end{array}$ & \multirow[t]{2}{*}{76.414} & \multirow[t]{2}{*}{0} & $\begin{array}{c}48.39 \\
\pm \\
16.35\end{array}$ & \multirow[t]{2}{*}{99.47} & \multirow[t]{2}{*}{0} & $\begin{array}{c}43.20 \\
\pm \\
15.95\end{array}$ & \multirow[t]{2}{*}{48.003} & \multirow[t]{2}{*}{0} & $\begin{array}{c}22.01 \\
\pm \\
6.09\end{array}$ & \multirow[t]{2}{*}{83.506} & \multirow[t]{2}{*}{0} \\
\hline & $(13-65)$ & & & $(11-55)$ & & & $(14-70)$ & & & $(14-70)$ & & & $(6-30)$ & & \\
\hline $\begin{array}{l}\text { Family } \\
\text { history } \\
\text { of } \\
\text { obesity }\end{array}$ & & \multirow{5}{*}{-3.124} & \multirow{5}{*}{$0.002^{*}$} & & \multirow{5}{*}{-3.03} & \multirow{5}{*}{$0.002^{*}$} & & \multirow{5}{*}{-2.533} & \multirow{5}{*}{$0.011^{*}$} & & \multirow{5}{*}{-0.021} & \multirow{5}{*}{$0.983^{*}$} & & & \\
\hline \multirow{2}{*}{$\begin{array}{l}\text { Obesity } \\
\text { in } \\
\text { family } \\
\text { history }\end{array}$} & $\begin{array}{c}47.13 \\
\pm \\
13.63\end{array}$ & & & $\begin{array}{l}37.04 \pm \\
12.75\end{array}$ & & & $\begin{array}{l}46.02 \\
\pm \\
16.58\end{array}$ & & & $\begin{array}{c}43.36 \\
\pm \\
15.35\end{array}$ & & & $\begin{array}{c}21.30 \\
\pm \\
5.92\end{array}$ & & \\
\hline & $(13-65)$ & & & $(11-55)$ & & & $(14-70)$ & & & $(14-70)$ & & & $(6-30)$ & -2.229 & $0.026^{*}$ \\
\hline $\begin{array}{l}\text { Absence } \\
\text { of } \\
\text { obesity }\end{array}$ & $\begin{array}{l}57.93 \\
\pm \\
14.74\end{array}$ & & & $\begin{array}{c}32.64 \pm \\
13.22\end{array}$ & & & $\begin{array}{c}50.34 \\
\pm \\
15.93\end{array}$ & & & $\begin{array}{c}43.07 \\
\pm \\
16.46\end{array}$ & & & $\begin{array}{c}22.59 \\
\pm \\
6.18\end{array}$ & & \\
\hline $\begin{array}{l}\text { family } \\
\text { history }\end{array}$ & $(14-65)$ & & & $(11-54)$ & & & $(14-70)$ & & & $(14-70)$ & & & $(8-30)$ & & \\
\hline
\end{tabular}




\begin{tabular}{|c|c|c|c|c|c|c|c|c|c|c|c|c|c|c|c|}
\hline $\begin{array}{l}\text { To Do } \\
\text { Some- } \\
\text { thing } \\
\text { Else } \\
\text { While } \\
\text { Eating }\end{array}$ & & \multirow{7}{*}{18.296} & \multirow{7}{*}{0} & & \multirow{7}{*}{10.287} & \multirow{7}{*}{0.006} & & \multirow{7}{*}{5.858} & \multirow{7}{*}{0.053} & & \multirow{7}{*}{1.805} & \multirow{7}{*}{0.406} & & \multirow{7}{*}{13.517} & \multirow{7}{*}{0.001} \\
\hline \multirow[t]{2}{*}{ Always } & $\begin{array}{c}43.79 \pm \\
15.71\end{array}$ & & & $\begin{array}{c}37.88 \\
\pm \\
13.58\end{array}$ & & & $\begin{array}{c}44.17 \\
\pm \\
18.43\end{array}$ & & & $\begin{array}{c}44.61 \\
\pm \\
16.33\end{array}$ & & & $\begin{array}{c}19.97 \\
\pm \\
6.90\end{array}$ & & \\
\hline & $(13-65)$ & & & $(11-54)$ & & & $(14-70)$ & & & $(14-70)$ & & & $(6-30)$ & & \\
\hline \multirow[t]{2}{*}{$\begin{array}{c}\text { Fre- } \\
\text { quently }\end{array}$} & $\begin{array}{c}52.01 \pm \\
10.49\end{array}$ & & & $\begin{array}{c}32.78 \\
\pm \\
12.13\end{array}$ & & & $\begin{array}{c}50.55 \\
\pm \\
13.60\end{array}$ & & & $\begin{array}{c}43.30 \\
\pm \\
15.21\end{array}$ & & & $\begin{array}{c}22.60 \\
\pm \\
5.62\end{array}$ & & \\
\hline & $(14-65)$ & & & $(11-54)$ & & & $(14-70)$ & & & (14-69) & & & $(9-30)$ & & \\
\hline \multirow[t]{2}{*}{ Never } & $\begin{array}{c}50.54 \pm \\
13.54\end{array}$ & & & $\begin{array}{c}33.89 \pm \\
13.98\end{array}$ & & & $\begin{array}{c}49.83 \\
\pm \\
17.52\end{array}$ & & & $\begin{array}{c}41.05 \\
\pm \\
16.85\end{array}$ & & & $\begin{array}{c}23.64 \\
\pm \\
5.05\end{array}$ & & \\
\hline & $(13-65)$ & & & $(11-55)$ & & & $(14-70)$ & & & $(14-70)$ & & & $(13-30)$ & & \\
\hline $\begin{array}{c}\text { Weekly } \\
\text { fast food } \\
\text { con- } \\
\text { sump- } \\
\text { tion }\end{array}$ & & \multirow{9}{*}{14.532} & \multirow{9}{*}{0.002} & & \multirow{9}{*}{7.886} & \multirow{9}{*}{0.048} & & \multirow{9}{*}{17.521} & \multirow{9}{*}{0.001} & & \multirow{9}{*}{11.963} & \multirow{9}{*}{0.008} & & \multirow{9}{*}{20.853} & \multirow{9}{*}{0} \\
\hline \multirow[t]{2}{*}{ Never } & $\begin{array}{c}50.64 \pm \\
10.31\end{array}$ & & & $\begin{array}{c}34.78 \\
\pm \\
12.54\end{array}$ & & & $\begin{array}{c}54.73 \\
\pm \\
14.57\end{array}$ & & & $\begin{array}{c}48.93 \\
\pm \\
15.17\end{array}$ & & & $\begin{array}{c}24.37 \\
\pm \\
5.43\end{array}$ & & \\
\hline & $(17-65)$ & & & $(12-52)$ & & & $(14-70)$ & & & $(14-70)$ & & & $(10-30)$ & & \\
\hline Once & $\begin{array}{c}52.26 \pm \\
11.73\end{array}$ & & & $\begin{array}{c}32.35 \\
\pm \\
12.38\end{array}$ & & & $\begin{array}{c}50.45 \pm \\
13.78\end{array}$ & & & $\begin{array}{c}39.84 \\
\pm \\
15.71\end{array}$ & & & $\begin{array}{c}22.72 \pm \\
5.67\end{array}$ & & \\
\hline & $(13-65)$ & & & $(12-54)$ & & & $(14-70)$ & & & (14-69) & & & $(9-30)$ & & \\
\hline \multirow[t]{2}{*}{ Twice } & $\begin{array}{c}45.35 \pm \\
15.28\end{array}$ & & & $\begin{array}{c}37.35 \\
\pm \\
13.99\end{array}$ & & & $\begin{array}{c}44.10 \\
\pm \\
18.83\end{array}$ & & & $\begin{array}{c}43.84 \\
\pm \\
14.85\end{array}$ & & & $\begin{array}{c}21.09 \\
\pm \\
6.42\end{array}$ & & \\
\hline & $(13-65)$ & & & $(11-54)$ & & & $(14-70)$ & & & $(18-70)$ & & & $(8-30)$ & & \\
\hline \multirow{2}{*}{$\begin{array}{l}\text { Three } \\
\text { and } \\
\text { more } \\
\text { times }\end{array}$} & $\begin{array}{c}46.53 \pm \\
15.09\end{array}$ & & & $\begin{array}{c}35.40 \\
\pm \\
13.70\end{array}$ & & & $\begin{array}{c}44.31 \\
\pm \\
16.85\end{array}$ & & & $\begin{array}{c}43.61 \\
\pm \\
16.97\end{array}$ & & & $\begin{array}{c}19.85 \\
\pm \\
6.15\end{array}$ & & \\
\hline & $(13-65)$ & & & $(11-55)$ & & & $(14-70)$ & & & $(14-70)$ & & & $(6-30)$ & & \\
\hline \multicolumn{16}{|l|}{$\begin{array}{l}\text { A Day in } \\
\text { Front of } \\
\text { a Screen } \\
\text { time }\end{array}$} \\
\hline \multirow{2}{*}{$\begin{array}{l}\text { No time- } \\
\text { con- } \\
\text { sum } \\
\text {-ing }\end{array}$} & $\begin{array}{c}54.21 \pm \\
10.72\end{array}$ & \multirow{6}{*}{6.569} & & $\begin{array}{c}30.14 \\
\pm \\
13.65\end{array}$ & & & $\begin{array}{c}59.11 \\
\pm \\
10.95\end{array}$ & & & $\begin{array}{c}42.75 \\
\pm \\
16.95\end{array}$ & & & $\begin{array}{c}24.82 \\
\pm \\
6.40\end{array}$ & & \\
\hline & $(23-65)$ & & & $(12-53)$ & & & $(15-70)$ & & & $(21-70)$ & & & $(12-30)$ & & \\
\hline $\begin{array}{l}\text { About an } \\
\text { hour }\end{array}$ & $\begin{array}{l}49.00 \pm \\
12.32\end{array}$ & & 0.037 & $\begin{array}{c}36.13 \\
\pm \\
12.40\end{array}$ & 7 & 0.03 & $\begin{array}{c}47.84 \\
\pm \\
15.59\end{array}$ & 14.21 & 0.001 & $\begin{array}{c}43.76 \\
\pm \\
15.20\end{array}$ & 0.334 & 0.846 & $\begin{array}{c}21.72 \\
\pm \\
5.89\end{array}$ & 7.55 & 0.023 \\
\hline & $(15-65)$ & & & $(11-54)$ & & & $(14-70)$ & & & $(14-70)$ & & & $(9-30)$ & & \\
\hline $\begin{array}{l}\text { Two and } \\
\text { more }\end{array}$ & $\begin{array}{c}48.02 \pm \\
15.68\end{array}$ & & & $\begin{array}{c}33.09 \\
\pm \\
14.05\end{array}$ & & & $\begin{array}{c}46.65 \\
\pm \\
17.86\end{array}$ & & & $\begin{array}{c}42.33 \pm \\
17.05\end{array}$ & & & $\begin{array}{c}21.82 \\
\pm \\
6.46\end{array}$ & & \\
\hline & $(13-65)$ & & & (11-54) & & & $(14-70)$ & & & $(14-70)$ & & & $(6-30)$ & & \\
\hline
\end{tabular}




\begin{tabular}{|c|c|c|c|c|c|c|c|c|c|c|c|c|c|c|c|}
\hline $\begin{array}{l}\text { Partic- } \\
\text { ipation } \\
\text { in sport/ } \\
\text { physical } \\
\text { activity }\end{array}$ & & \multirow{9}{*}{9.767} & \multirow{9}{*}{0.021} & & \multirow{9}{*}{9.71} & \multirow{9}{*}{0.021} & & \multirow{9}{*}{12.72} & \multirow{9}{*}{0.005} & & \multirow{9}{*}{2.053} & \multirow{9}{*}{0.562} & & \multirow{9}{*}{7.174} & \multirow{9}{*}{0.067} \\
\hline \multirow{2}{*}{$\begin{array}{c}\text { No } \\
\text { partici- } \\
\text { pation }\end{array}$} & $\begin{array}{c}45.09 \\
\pm \\
16.14\end{array}$ & & & $\begin{array}{c}37.30 \\
\pm \\
13.30\end{array}$ & & & $\begin{array}{c}45.00 \\
\pm \\
16.25\end{array}$ & & & $\begin{array}{c}43.66 \\
\pm \\
14.94\end{array}$ & & & $\begin{array}{c}21.05 \\
\pm \\
6.30\end{array}$ & & \\
\hline & $(13-64)$ & & & $(11-55)$ & & & $(14-70)$ & & & $(14-67)$ & & & $(9-30)$ & & \\
\hline \multirow[t]{2}{*}{$\begin{array}{c}1 \text { time } \\
\text { per week }\end{array}$} & $\begin{array}{c}49.47 \\
\pm \\
11.98\end{array}$ & & & $\begin{array}{c}34.72 \\
\pm \\
12.12\end{array}$ & & & $\begin{array}{c}49.52 \\
\pm \\
15.73\end{array}$ & & & $\begin{array}{c}43.99 \\
\pm \\
16.06\end{array}$ & & & $\begin{array}{c}21.80 \\
\pm \\
5.80\end{array}$ & & \\
\hline & $(14-65)$ & & & $(12-54)$ & & & $(14-70)$ & & & $(14-70)$ & & & $(6-30)$ & & \\
\hline \multirow[t]{2}{*}{$\begin{array}{c}2-3 \text { times } \\
\text { a week }\end{array}$} & $\begin{array}{c}51.63 \\
\pm \\
11.14\end{array}$ & & & $\begin{array}{c}32.43 \\
\pm \\
13.35\end{array}$ & & & $\begin{array}{c}49.12 \\
\pm \\
16.28\end{array}$ & & & $\begin{array}{c}41.54 \\
\pm \\
16.86\end{array}$ & & & $\begin{array}{c}22.53 \\
\pm \\
6.08\end{array}$ & & \\
\hline & $(13-65)$ & & & $(11-52)$ & & & $(14-70)$ & & & $(14-70)$ & & & $(8-30)$ & & \\
\hline \multirow[t]{2}{*}{$\begin{array}{c}4 \text { times a } \\
\text { week }\end{array}$} & $\begin{array}{c}53.54 \\
\pm \\
10.18\end{array}$ & & & $\begin{array}{c}32.51 \\
\pm \\
13.32\end{array}$ & & & $\begin{array}{c}54.46 \\
\pm \\
15.14\end{array}$ & & & $\begin{array}{c}45.00 \\
\pm \\
16.25\end{array}$ & & & $\begin{array}{c}23.89 \\
\pm \\
5.68\end{array}$ & & \\
\hline & $(22-65)$ & & & $(11-55)$ & & & $(14-70)$ & & & $(14-70)$ & & & $(9-30)$ & & \\
\hline
\end{tabular}

*Mann Whitney-U testi, ${ }^{* \star K}$ Kruskal Wallis Testi

Findings showed that the total score of DEAS, a healthy dietexercise behaviour and meal scheme DEBS subscales increased with the following features: High BMI $(p<0.001)$, more than revenue expense $(p<0.050)$, absence of obesity in the family history $(p<0.050)$, frequently to do something else while eating $(p<0.050)$, eat fast food once per week $(p<0.050)$, any time spent in front of a screen $(\mathrm{p}<0.050)$, take part in sports/physical activity regularly ( 4 times a week) $(\mathrm{p}<0.050)$. The mean subscales scores for the entire study and levels of significance are given in Table 4 .

\section{Discussion}

The assessment of the eating attitudes can be useful to understand people's relationship with the foods and their associated behavioural choices. Diet and physical activity evaluation of the DEAS total score and the DEBS subscales mean scores were performed on midwifery students. The findings of this study reported a significant relationship between dietphysical activity attitudes and behaviours [4,11]. There is a significant effect of the DEAS total score and the DEBS subscales mean scores on psychological eating and unhealthy diet-exercise behaviours; the students who have high DEAS total scores, they have less psychological eating and unhealthy diet-exercise behaviours. In addition, in the agreement with the current study, the mean scores of the high healthy diet-exercise behaviour and meal scheme were also found among university students who had high DEAS total scores. The findings of this study reported that the diet-exercise attitude was strong correlated with diet-exercise behaviour (psychological eating, healthy diet-exercise behaviour and meal scheme). The similar studies also support this finding of the present study [12-16].
The findings of this study reported a significant relationship between Socio-demographic, diet, physical activity characteristics and diet-exercise behaviour. This study underlined that the values of the underweight, overweight and obese participants were high and as the values increased, the psychological eating and unhealthy diet-exercise behaviours increased. Also, the participants who had the absence of obesity in family history showed the more evidences of improved diet-exercise behaviour. We found that the participants, who frequently do something else while eating and eat fast food once per week, are more sedentary (a lot of time spent in front of a screen) or less physically active. Since the participants who do something else while eating, have weekly fast food consumption and spend time in front of screen may be associated with an increased risk for the psychological eating and unhealthy diet-exercise behaviours. Our findings were similar to previous studies [6,17-21]. Given the importance of the university years in establishing lifelong dietary habits [22], it is imperative to have better understanding of diet-exercise attitude and behaviour of the university students. The maintaining healthy habits, including reducing sedentary behaviour and improving physical activity, is essential even in the fit person.

\section{Conclusion}

Diet-exercise attitudes and behaviours were moderate and affected by some demographic characteristics. The results of this study highlight the diet-exercise behaviours and attitudes of the midwifery students, and also identifying and assessing interventions that target reducing sedentary life, establishing the healthy attitude and behaviour for the midwifery students. To maximize physical wellness, we may obtain the greatest potential 
benefit based on reducing sedentary life and establishing the healthy attitude and behaviour by providing a foundation for a lifetime of positive behaviours. It is considered that students should be encouraged to about regular and balanced diet-exercise. A better understanding of the factors affecting the dietary patterns of future health professionals can help improve the quality of diet, which has significant implications for the overall reduction of chronic disease risk.

\section{References}

1. Kabir A, Miah S, Islam A (2018) Factors influencing eating behavior and dietary intake among resident students in a public university in Bangladesh: A qualitative study. PIOS ONE 13(6): e0198801.

2. Waxman A (2004) WHO global strategy on diet, physical activity and health. Food Nutr Bull 25(3): 292-302.

3. Plotnikoff RC, Costigan SA, Kennedy SG, Robards SL, Germov J, et al (2018) Efficacy of interventions targeting alcohol, drug and smoking behaviors in university and college students: A review of randomized controlled trials. J Am Coll Health 13: 1-17.

4. Pengpid S, Peltzer K (2015) Dietary health behaviour and beliefs among university students from 26 low, middle and high income countries. Asia Pac J Clin Nutr 24(4): 744-752.

5. Sepulveda AR, Carrobles JA, Gandarillas AM (2008) Gender, school and academic year differences among Spanish university students at highrisk for developing an eating disorder: An epidemiologic study. BMC Public Health 8: 102.

6. Ludy MJ, Crum A, Young C, Morgan A, Tucker R (2018) First-Year University Students Who Self-Select into Health Studies Have More Desirable Health Measures and Behaviors at Baseline but Experience Similar Changes Compared to Non-Self-Selected Students. Nutrients 10(3): 362 .

7. Seppälä T, Hankonen N, Korkiakangas E, Ruusuvuori J, Laitinen J (2017) National policies for the promotion of physical activity and healthy nutrition in the workplace context: a behaviour change wheel guided content analysis of policy papers in Finland. BMC public health 18(1): 87

8. Hills AP, King NA, Armstrong TP (2007) The contribution of physical activity and sedentary behaviours to the growth and development of children and adolescents. Sports med 37(6): 533-545.

9. Mc Neill J, Doran J, Lynn F, Anderson G, Alderdice F (2012) Public health education for midwives and midwifery students: a mixed methods study. BMC Pregnancy and Childbirth 12: 142.

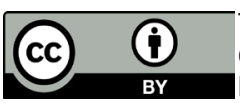

This work is licensed under Creative

Commons Attribution 4.0 License

DOI: 10.19080/JOJNHC.2019.10.555780
10. Kalay R, Sonay Turkmen A (2015) Factors Affectıng the Nutrıtıon and Exercise Behaviours of Adolescents. International Refereed Journal of Nutrition Research 5: 1-14.

11. Psouni S, Chasandra M, Theodorakis Y (2016) Patterns of Eating and Physical Activity Attitudes and Behaviors in Relation to Body Mass Index. Psychology 7(2): 180-192.

12. Akgul Gundogdu N, Sevig EU, Guler N (2018) The effect of the solutionfocused approach on nutrition-exercise attitudes and behaviours of overweight and obese adolescents: Randomised controlled trial. J Clin Nurs 27(7-8): e1660-e1672.

13. Valladares M, Peña, D, Pino C (2018) Psychological aspects associated with eating behaviour and obesity in university Chilean students. Clinical Nutrition 37: S208.

14. Alvarenga MS, Pereira RF, Scagliusi FB, Philippi ST, Estima CCP, et al. (2010) Psychometric evaluation of the Disordered Eating Attitude Scale (DEAS). English version. Appetite 55(2): 374-376.

15. Torres SJ, Nowson CA (2007) Relationship between stress, eating behavior, and obesity. Nutrition 23(11-12): 887-894.

16. Allison KC, Grilo CM, Masheb RM, Stunkard AJ (2005) Binge Eating Disorder and Night Eating Syndrome: A Comparative Study of Disordered Eating. J Consult Clin Psychol 73(6): 1107-1115.

17.Xu F, Greaney ML, Cohen SA, Riebe D, Greene GW (2018) The Association between Adolescent's Weight Perception and Health Behaviors: Analysis of National Health and Nutrition Examination Survey Data, 2011-2014. Journal of Obesity 2018: 1-8.

18. Zhang C, Zheng X, Huang H, Su C, Zhao H, et al. (2018) A Study on the Applicability of the Health Action Process Approach to the Dietary Behavior of University Students in Shanxi, China. J Nutr Educ Behav 50(4): 388.e1-395.e1.

19. Peterson NE, Sirard JR, Kulbok PA, DeBoer MD, Erickson JM (2018) Sedentary behavior and physical activity of young adult university students Res Nurs Health 41(1): 30-38.

20. Kyrkou C, Tsakoumaki F, Fotiou M, Dimitropoulou A, Symeonidou M, et al. (2018) Changing Trends in Nutritional Behavior among University Students in Greece, between 2006 and 2016. Nutrients 10(1): E64.

21. Fransen HP, May AM, Beulens JWJ, Struijk EA, de Wit GA, et al. (2014) Association between Lifestyle Factors and Quality-Adjusted Life Years in the EPIC-NL Cohort. PLoS One 9(11): e111480.

22. Mueller M, Blondin S, Korn A, Bakun, P, Tucker K, et al. (2018) Behavioral Correlates of Empirically-Derived Dietary Patterns among University Students. Nutrients 10(6): E716.

\section{Your next submission with Juniper Publishers will reach you the below assets}

- Quality Editorial service

- Swift Peer Review

- Reprints availability

- E-prints Service

- Manuscript Podcast for convenient understanding

- Global attainment for your research

- Manuscript accessibility in different formats

( Pdf, E-pub, Full Text, Audio)

- Unceasing customer service

Track the below URL for one-step submission https://juniperpublishers.com/online-submission.php 